[0212-7199 (2005) 22: 1; pp. 39-42] ANALES DE MEDICINA INTERN Copyright (C) 2005 ARAN EDICIONES, S.L

AN. MED. INTERNA (Madrid) Vol. 22, N. ${ }^{\circ} 1$, pp. $39-42,2005$

\title{
Valor terapéutico añadido de los medicamentos: ¿qué es, cómo se evalúa y cuál debería ser su papel en política farmacéutica?
}

\author{
J. SOTO ÁLVAREZ
}

Departamento de Farmacoepidemiología \& Farmacoeconomía. Unidad Médica. Pfizer S.A. Alcobendas. Madrid

DRUG ADDED THERAPEUTIC VALUE: WHAT IS, HOW IS IT ASSES SED AND WHAT SHOULD BE ITS ROLE IN PHARMACEUTICAL POLICY?

Soto Álvarez. J. Valor terapéutico añadido de los medicamentos: ¿qué es, cómo se evalúa y cuál debería ser su papel en política farmacéutica? An Med Interna (Madrid) 2005; 22: 39-42.

\section{INTRODUCCIÓN}

Al inicio del siglo XXI, España dispone de un sistema sanitario equitativo y cohesionado, con una cobertura universal y una financiación pública en su gran mayoría, y de una gran calidad, lo que ha permitido que se haya alcanzado un elevado nivel de salud en la población. A este hecho ha contribuido de manera importante la síntesis, desarrollo y comercialización de nuevos medicamentos, al incrementar la expectativa de vida de la población con importantes mejoras en su calidad de vida (1).

Ahora bien, el entorno de la atención sanitaria se ha complicado mucho en los últimos años. Por una parte, cada vez es más notorio la inversión de la pirámide poblacional, por lo que cada vez hay una mayor prevalencia de sujetos ancianos, y cada vez hay más pacientes que viven más tiempo con sus enfermedades de manera crónica. Por otra parte, en los últimos años hemos asistido a una llegada masiva de inmigrantes, los cuales demandan un cuidado sanitario importante, dadas sus carencias en salud. Además, la sociedad en su conjunto cada vez presenta una mayor educación sanitaria y es más consciente de su esfuerzo económico (a través de los impuestos) para financiar la atención sanitaria, y por lo tanto, cada vez es más demandante en servicios y prestaciones sanitarias de calidad.

Sin embargo, los recursos en sanidad siguen siendo insuficientes, ya que la atención sanitaria está en continua y cons- tante evolución, y la presión y demanda asistencial crecen sin precedentes y sin ningún tipo de control.

De esta manera, en estos momentos las autoridades sanitarias tienen que atender y dar cobertura a necesidades cada vez más elevadas de la sociedad en materia sanitaria con recursos que crecen a una velocidad mucho más baja y, que por lo tanto, cada vez son más limitados para poder cubrir las crecientes demandas sanitarias. Este hecho ha motivado la necesidad de gestionar los recursos existentes de una forma cada vez más eficaz y eficiente (2).

En este contexto, la factura farmacéutica se ha ido incrementando progresivamente en la última década, aunque de manera proporcionada al Producto Interior Bruto y al gasto sanitario total de nuestro país (3). Con el fin de intentar controlarla y mantenerla dentro de unos límites, se han establecido diversas medidas estando dirigidas, por una parte, a controlar la oferta de medicamentos disponibles (potenciación de genéricos, implantación de precios de referencia, elaboración de listas positivas y negativas, introducción de visados, negociación del precio y nivel de financiación de los nuevos medicamentos y de las nuevas indicaciones), y por otro lado, a gestionar la demanda de medicamentos y a fomentar un uso racional de los mismos, tanto por parte de los facultativos como por parte de los pacientes (elaboración de formularios y guías terapéuticas, implantación de sistemas de prescripción electrónicos, creación de programas formativos e informativos, etc.).

Tradicionalmente, dentro de la Unión Europea, para que un nuevo medicamento fuera aprobado para su comerciali-

Trabajo aceptado: 9 de septiembre de 2004

Correspondencia: Javier Soto Álvarez. Unidad Médica. Pfizer S.A. Avda. de Europa 20-B. Parque Empresarial de La Moraleja. 20108 Alcobendas (Madrid). e-mail: javier.soto.alvarez@pfizer.com 
zación, solamente se necesitaba que demostrara un nivel de calidad, seguridad y eficacia, similar o mayor que otras alternativas terapéuticas presentes en el mercado. Sin embargo, en el año 2000 el Consejo de la Unión Europea, dentro de sus conclusiones sobre medicamentos y salud pública, subrayó la importancia de la identificación de medicamentos con un destacado valor terapéutico añadido (4). Muy recientemente, el Parlamento Europeo y el Consejo de la Unión Europea, a través del Reglamento $\mathrm{n}^{\circ}$ $726 / 2004$, han instado a los Estados miembros a determinar el beneficio terapéutico aportado por cada nuevo medicamento (donde el valor terapéutico añadido es una dato crucial), con el fin de permitir en todo momento la reevaluación de la relación beneficio-riesgo de un medicamento (5).

Por otra parte, en los últimos tiempos se ha puesto de manifiesto la importancia creciente que se otorga al valor terapéutico de los medicamentos en contextos bien diferentes. Así, en fechas recientes la ministra de sanidad ha especificado que "sólo los medicamentos que tengan realmente valor terapéutico serán financiados por el Sistema Nacional de Salud (SNS)" (6), y en otro foro diferente, dónde fueron evaluadas las funciones del comité mixto de evaluación de nuevos medicamentos (organismo creado recientemente por Cataluña, País vasco y Andalucía), se especificó que su misión va a ser evaluar la aportación terapéutica de los nuevos medicamentos, valorando el valor terapéutico añadido de éstos con respecto a las opciones ya disponibles en el mercado (7).

En este contexto, las preguntas claves que deberíamos hacernos son: ¿qué es el valor terapéutico añadido?, ¿cómo se puede medir y evaluar?, ¿cuál debería ser su papel a la hora de diseñar una política farmacéutica racional?

Para poder contestar a la primera pregunta, es necesario dejar bien claro que los datos de eficacia, seguridad y calidad se refieren a los beneficios terapéuticos de un medicamento en las condiciones ideales del ensayo clínico controlado (selección estricta de pacientes, seguimiento exhaustivo de los mismos, ausencia de la población de mayor riesgo: ancianos, polimedicados y pacientes con varias co-morbilidades asociadas, etc.), pero no nos dicen nada en relación a otros posibles efectos beneficiosos del nuevo medicamento en la práctica médica diaria, tales como las ventajas en calidad de vida o en satisfacción con el tratamiento, el incremento en las preferencias de los pacientes, el aumento del cumplimiento terapéutico y la persistencia del tratamiento, los resultados clínicos obtenidos en condiciones de práctica habitual (o sea, la efectividad clínica), las posibles ventajas en eficiencia (esto es, en la relación coste/efectividad), las ventajas en términos de morbi-mortalidad, así como posibles ventajas en productividad laboral y en el nivel de discapacidad de los pacientes en relación a su enfermedad.

Todas estos parámetros y datos que miden y evalúan variables y aspectos diferentes de la eficacia, seguridad y calidad, es lo que se denomina valor terapéutico añadido, y su conocimiento y valoración es totalmente complementaria (y tan importante) como los datos de eficacia, seguridad y calidad de los nuevos medicamentos, y podrían ayudar, tremendamente, a tomar decisiones acertadas en política de medicamentos.

Si queremos responder a la segunda pregunta, va a ser necesario definir con criterios objetivos, explícitos y relevantes cuándo se va a considerar que un valor terapéutico añadido va a ser elevado e importante, cuando se compara con opciones ya existentes en el mercado. A modo de ejemplo, se podría considerar que el valor terapéutico añadido fuera significativo y relevante siempre que el cambio o mejoría en calidad de vida o satisfacción del paciente presentase un tamaño del efecto mayor de 0,8 y un número necesario a tratar (NNT) menor de 10; cuando se considerase altamente coste-efectivo, (esto es, presentase un coste por año de vida ganado menor de $18.000 \quad$ ); siempre que el incremento en la tasa de cumplimiento terapéutico fuese superior al 20\%; cuando la reducción absoluta de la tasa de mortalidad fuera superior al $5 \%$, con un NNT por debajo de 20 , etc.

Para contestar a la tercera pregunta, será necesario decidir cómo va a ser incorporado el valor terapéutico añadido de los nuevos medicamentos (y cuál va a ser su peso específico) a la hora de elaborar y establecer una política farmacéutica coherente y racional, de tal manera que este dato se tenga en cuenta en la toma de decisiones que afecten a los medicamentos:

-Fijación de precio y nivel de financiación de los nuevos medicamentos.

- Su inclusión en los formularios y guías terapéuticas.

- Su incorporación en las recomendaciones incluidas en los boletines terapéuticos y en los sistemas electrónicos de prescripción.

La negociación del precio y la fijación del nivel de financiación de los nuevos medicamentos y/o nuevas indicaciones, es una potestad del Ministerio de Sanidad y Consumo, a través de la Dirección General de Farmacia y Productos Sanitarios, con la participación activa de la Agencia Española de Medicamentos y Productos Sanitarios (a través de la emisión de recomendaciones sobre los productos, en cuanto a su nivel de eficiencia y el grado de valor terapéutico que añade al arsenal terapéutico ya existente), tal y como se recoge en la Ley 16/2003, de 28 de Mayo", de cohesión y calidad del sistema nacional de salud" (8) y en el Real Decreto 1555/2004, de 25 de Junio, "por el que se establece la estructura orgánica básica del Ministerio de Sanidad y Consumo" (9).

En la actualidad, la negociación del precio de un nuevo medicamento se basa en el Real Decreto 271/1990, de 23 de Febrero, "sobre la reorganización de la intervención de precios de las especialidades farmacéuticas de uso humano" (10), mientras que la fijación del nivel de financiación se rige por la Ley 25/1990, de 20 de Diciembre, " del Medicamento" (11) y el Real Decreto 83/1993, de 22 de Enero, "por el que se regula la selección de Medicamentos a efectos de su financiación por el sistema nacional de salud" (12). En estas normativas, ya se daba entrada (aunque de una manera muy tímida) a la posibilidad de incorporar a las decisiones de precio y financiación, el valor terapéutico añadido de los nuevos medicamentos frente a las opciones ya existentes, a través de lo que se denomina como "utilidad terapéutica y social aportada por el nuevo producto" y la "existencia de medicamentos ya disponibles a menor precio o inferior costo de tratamiento".

El hecho real es que, desde su publicación hasta el momento actual, la inclusión del valor terapéutico añadido de los medicamentos en la negociación del precio y del nivel de financiación ha sido escasa y siempre como un valor secundario y totalmente voluntario, sin que se hayan 
establecido unos criterios objetivos y explícitos sobre su utilidad y aplicación real.

Dado que el valor terapéutico añadido es un dato complementario a los datos de eficacia y seguridad, y puede ser de gran ayuda en la toma de decisiones, debería ser tenido en cuenta en el proceso de fijación del precio y nivel de financiación de un nuevo medicamento, de tal manera que a mayor valor terapéutico añadido (frente a las opciones ya existentes), mayor precio debería obtener y mejor tratamiento en la fijación del nivel de financiación debería tener, ya que esto significa que el nuevo medicamento aporta valores adicionales que se van a traducir en una mejor calidad asistencial y en lograr mejores resultados en salud.

Los formularios y las guías terapéuticas son excelentes herramientas para incrementar el uso racional de los medicamentos y garantizar la utilización rutinaria de aquéllos que más evidencia científica han demostrado y que más ventajas van a proporcionar a los pacientes y a la sociedad de manera global.

Cuando se revisa el panorama actual en nuestro país en relación a los formularios y guías terapéuticas existentes, se puede constatar que existen muchas, siendo auspiciadas por distintos organismos (sociedades científicas, autoridades sanitarias de las comunidades autónomas, grupos de profesionales sanitarios, etc.), que son de muy desigual calidad y que han sido elaboradas siguiendo metodologías muy dispares y desiguales, y sin que hayan sido evaluadas por nadie en relación a unos estándares mínimos de calidad.

Además, cuando se revisa la información que se evalúa en su diseño, solamente los datos de eficacia, seguridad y tolerabilidad son tenidos en cuenta junto con el coste/día de los medicamentos evaluados, sin que se tengan en cuenta para nada otros datos, tales como el valor terapéutico añadido de los diferentes medicamentos existentes (raramente son evaluados datos de calidad de vida y/o satisfacción con el tratamiento, o resultados de estudios coste-efectividad, o datos de cumplimiento terapéutico, etc.), datos de gran interés a la hora de decidir que incluir en los formularios y guías terapéuticas.

Los sistemas de prescripción electrónicos, más conocidos como la receta electrónica, son instrumentos en proceso de implantación en la mayoría de los servicios regionales de salud de nuestro país, en un intento por gestionar la demanda de medicamentos en atención primaria e incrementar un uso racional de los mismos (13).

El objetivo de estos sistemas es que cuando el médico vaya a prescribir un medicamento a un paciente con una determinada enfermedad, el sistema le aconseje escoger entre un grupo de fármacos que se recomiendan por defecto, y si el médico entiende que es necesario prescribir otro, deberá explicar con detalle el motivo de su elección y que nuevo medicamento va a prescribir.
Un gran peligro es que a la hora de decidir qué medicamentos se recomiendan para tratar las distintas patologías, solamente se tengan en cuenta criterios economicistas y de contención de costes, incluyéndose sistemáticamente aquellas opciones terapéuticas que presenten un menor coste/día, sin que se tenga en cuenta en la elección el valor terapéutico añadido e innovador de los medicamentos, y por lo tanto, sus posibles ventajas en calidad de vida, satisfacción con el tratamiento, eficiencia, etc.

En estos momentos, es necesario elaborar propuestas coherentes e implantar medidas estructurales en nuestro SNS para poder garantizar que los medicamentos que aporten verdadero valor terapéutico añadido a la sociedad y al sistema, y que realmente son innovadores, estén disponibles para aquellos pacientes que los necesiten y sean los que se empleen rutinariamente en la práctica médica diaria.

El beneficio va a ser poder disponer de un SNS que promueve el uso de los medicamentos que mejores resultados en salud van a producir, lo que incrementará la calidad asistencial del mismo y elevará en nivel de salud de los ciudadanos de nuestro país, fin último de todo sistema sanitario. Está claro que de poco nos va a servir disminuir la factura farmacéutica globalmente sin más, si es a consta de disminuir la calidad asistencial de los pacientes y de mermar el control de sus patologías, ya que antes o después se producirá un efecto rebote, que motivará un incremento sustancial del gasto sanitario, derivado de tratar el mal control y las recidivas de los pacientes.

En la actualidad, es necesario buscar en nuestro país un sincretismo entre la equidad y el acceso de los ciudadanos al sistema sanitario y la sostenibilidad y la viabilidad del mismo, de tal manera que solamente se promueva el uso de aquellas intervenciones terapéuticas que vayan a incrementar los resultados en salud de la sociedad, y que por lo tanto vayan a ser eficientes. Los medicamentos innovadores y que aportan valor terapéutico añadido entran dentro de este grupo, y por lo tanto debería fomentarse su utilización de forma rutinaria.

Tanto las autoridades sanitarias centrales a nivel del Ministerio de Sanidad, responsables de controlar y manejar la oferta de los medicamentos en nuestro país, como las autoridades sanitarias a nivel autonómico, responsables de gestionar la demanda de los ciudadanos en materia de medicamentos y de propiciar un uso racional de los mismos, deberían fomentar y facilitar la incorporación de los medicamentos innovadores y con un elevado valor terapéutico añadido al arsenal terapéutico de nuestro país, derecho inherente de los ciudadanos como copropietarios y accionistas del SNS de nuestro país. Por lo tanto, deberían incorporar a sus procedimientos y normativas la necesidad de fomentar e impulsar aquellos medicamentos que ofrezcan valores terapéuticos añadidos y sean innovadores y, posteriormente, promover su utilización en la práctica asistencial.

\section{Bibliografía}

1. Soto J, Olivella P. ¿Son los medicamentos una inversión económicamente rentable para el sistema nacional de salud en España? Med Clin (Barc) 2001; 117: 622-624.

2. López Casasnovas G. Racionalizar y optimizar el gasto sanitario, clave de la aportación de la economía de la salud al bienestar social. En: Farmacoeconomía e Investigación de Resultados en la Salud: Principios y Práctica. Coordinadotes: Domínguez-Gil Hurlé A, Soto Álvarez J. Real Academia Nacional de Farmacia. Madrid 2002. 
3. Simó Miñana J, de Pablo González R, Ramos Maestre MJ, Gaztambide Ganuza MS. ¿Gastamos demasiado en medicamentos? El gasto farmacéutico, un bien de primera necesidad entre los países europeos. Aten Primaria 2004; 33: 244-253.

4. Diario Oficial del Consejo, 218 de 31 de Julio de 2000

5. Diario Oficial de la Unión Europea. Reglamento (CE) N $N^{\circ} 726 / 2004$ del Parlamento Europeo y del Consejo de 31 de Marzo de 2004

6. Sanidad anuncia una nueva política farmacéutica más restrictiva para antes de fin de año. JANO on-line. 17/06/2004 (http://db.doyma.es/cgibin/wdbcgi.exe/doyma/press.archivo) (Accesibilidad verificada el 26 de Julio de 2004)

7. El valor de los nuevos fármacos, a examen. Cataluña, País Vasco y Andalucía informan a los médicos de la aportación terapéutica de los nuevos productos. El Global N ${ }^{\circ}$ 208, 5 al 11 de Julio de 2004. www.elglobal.net (Accesibilidad verificada el 26 de Julio de 2004).
8. Ley $16 / 2003$, de 28 de mayo, de cohesión y calidad del Sistema Nacional de Salud. BOE del 29 de mayo de 2003.

9. Real Decreto $1555 / 2004$, de 25 de junio, por el que se desarrolla la estructura orgánica básica del Ministerio de Sanidad y Consumo. BOE de 26 de junio de 2004.

10. Real Decreto $217 / 1990$, de 23 de febrero, sobre la reorganización de la intervención de precios de las especialidades farmacéuticas de uso humano. BOE de 2 de marzo de 1990.

11. Ley 25/1990, de 20 de diciembre, del Medicamento. BOE de 22 de diciembre de 1990.

12. Real Decreto 83/1993, de 22 de enero, por el que se regula la selección de los medicamentos a efectos de su financiación por el Sistema Nacional de Salud. BOE de 19 de febrero de 1993.

13. Schiff GD, Rucker D. Computerized prescribing. Building the electronic infrastructure for better medication usage. JAMA 1998; 279: 1024-1029. 